POSTABSORPTIVE GLUCOSE, TASTE AND INGESTION 


\title{
POSTABSORPTIVE GLUCOSE DECREASES THE EXCITATORY EFEECTS OF TASTE ON INGESTION
}

\section{By}

Michel Bédard

\begin{abstract}
A Thesis
Submitted to the School of Graduate Studies

in Partial Fulfilment of the Requirements

for the Degree

Master of Science
\end{abstract}

(C) Copyright by Michel Bédard, December 1989 
MASTER OF SCIENCE (1989)

(Psychology)

TITLE :

AUTHOR :

SUPERVISOR:

NUMBER OF PAGES: vi, 44
MCMASTER UNIVERSITY

Hamilton, Ontario

Postabsorptive Glucose Decreases the Excitatory Effects of Taste on Ingestion

Michel Bédard, B.A. (Concordia University)

Dr. H.P. Weingarten 
Abstract

To test the hypothesis that postprandial rises of plasma glucose attenuate the motivation derived from positive tastes, I analyzed the effects of intraperitoneal (ip) injections of glucose on sham feeding, a preparation in which food intake is motivated primarily by taste sensations. IP glucose suppressed sham feeding, with maximal suppressions approximating 42\%, but only when glucose was administered contiguous with oropharyngeal stimulation. The food intake inhibition produced by ip glucose interacted with palatability; smaller doses of glucose were required to suppress less concentrated sucrose solutions. Closing the gastric cannula increased the potency with which ip glucose inhibited eating suggesting synergy of postabsorptive glucose with other postgastric satiety signals. The inhibition of eating produced by ip glucose did not result from malaise. Thus, postabsorptive rises of plasma glucose decrease the ability of taste cues to drive ingestion and suggest that this phenomenon may contribute to spontaneous meal termination. 


\section{Acknowledgments}

I would like to thank my supervisor, Dr. Harvey P. Weingarten, for his support and guidance. Harvey is enthusiastic and deeply committed to research. He has helped me develop research, analytical and writing skills beyond what I could have expected. I would also like to thank the other committee members, Dr. Allan and Dr. Seigel.

My stay in graduate school would not have been such a wonderful experience if it was not for the friendship and support of numerous individuals. It is impossible to name everyone but some merit a special mention. First, I wish to express my deepest gratitude to my highly respected colleague and friend Bruce Weaver. Bruce has made the last three years appear like three months; my only regret is that we did not meet earlier. Second, I would like to thank my graduate student colleagues, especially Rochelle Cole, Elaine MacNiven, Cheryl McCormick and Mike Mosher (John, Duke, Otto, Fritz, Heinie, Onan I, the other musketeer). Third, my lab colleagues also deserve my gratitude for their help and support. Among them Kent Conover merits a special mention for the endless hours spent together discussing research. Finally, I thank Lori for her encouragement and presence. She is one more excellent reason to say "Life is Beautiful".

I dedicate this thesis to my parents. 
Table of Contents

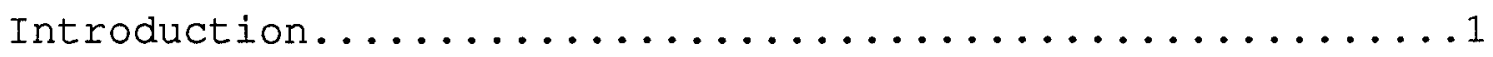

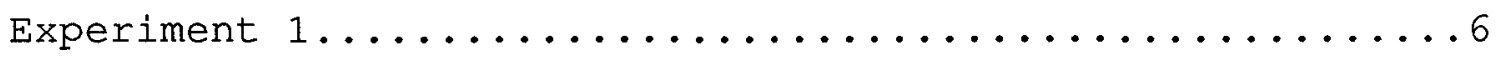

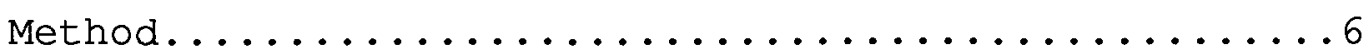

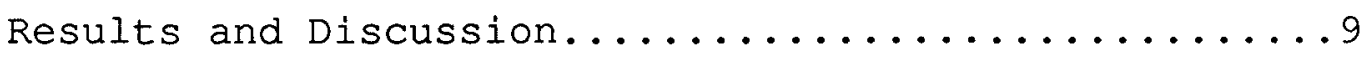

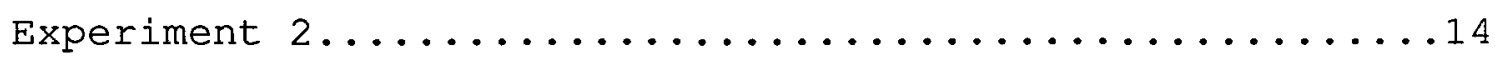

Method, Results and Discussion..............14

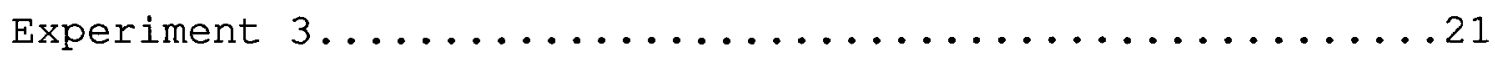

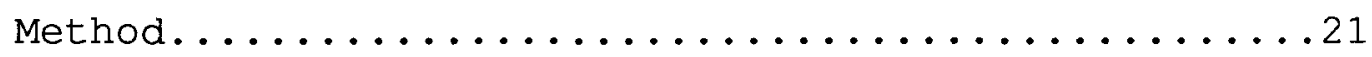

Results and Discussion.....................

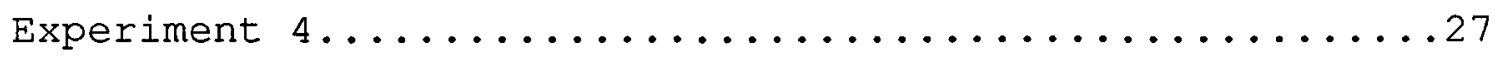

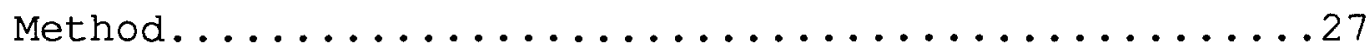

Results and Discussion................... 8

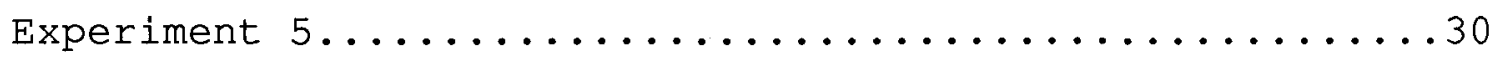

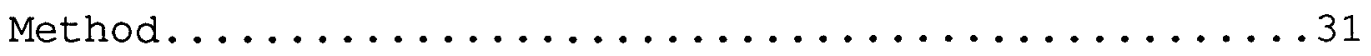

Results and Discussion.....................

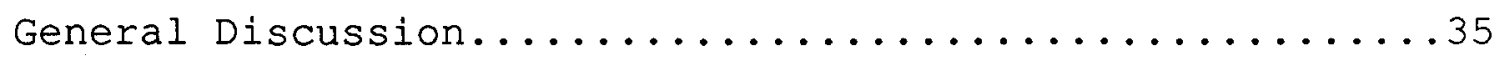

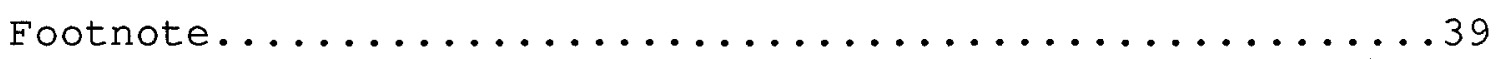

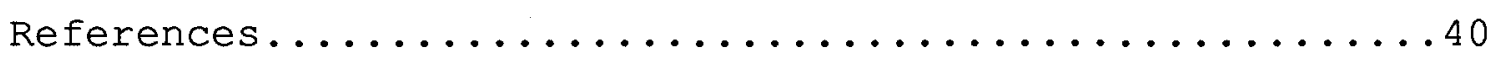


List of Tables and Figures

Table 1 Protocol for Experiment $1 \ldots \ldots \ldots \ldots \ldots \ldots$

Figure 1 Amount of 15\% Sucrose Sham Fed.............11

Figure 2 Water and Food Intake after D-Glucose.......17

Figure 3 Amount of 15\% Sucrose Sham Fed after

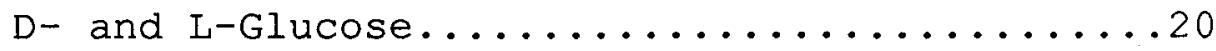

Table 2 Temporal Parameters and Intake Suppression...22

Figure 4 Percent Suppression after $1.5 \mathrm{mg} / \mathrm{kg} \ldots \ldots \ldots 25$

Table 3 Effect of ip Glucose on Sham Feeding of

Different Sucrose Concentrations...........29

Figure 5 Amount of 15\% Sucrose Normal Fed...........33 
INTRODUCTION

Grill and Berridge (1985) suggested that a food's "palatability" is comprised of three components: 1) the sensory properties of the food, 2) associations of the taste with positive or negative postingestive consequences, and 3 ) the internal state of the organism. The influence of the sensory properties of food, especially of sweet taste, on meal size has been subject to considerable analysis (Cagan \& Maller, 1974; Davis, 1973; Weingarten \& Watson, 1982; Young, 1967). To isolate the role of a food's sensory properties on intake, procedures such as brief-encounter tests (Christensen, 1961; Young, 1967) and sham feeding (Davis \& Campbell, 1973; Mook, 1963; Weingarten \& Watson, 1982) are used to minimize changes in the internal state of the organism. There has also been considerable research examining the effects on intake of taste associations with positive (Booth, 1972, 1985) or negative (Garcia, Hankins \& Rusiniak, 1974; Riley \& Tuck, 1985) postingestive consequences. The present thesis is concerned with the third component of palatability, the role of internal factors.

Although it is obvious that internal state influences eating, few have looked directly at how the internal state of the organism interacts with the processing of taste 
information. Since palatability is a major determinant of meal size (Davis \& Levine, 1977), and internal state changes over the course of a meal, examination of the interaction between internal state and taste may provide insight into mechanisms of meal termination.

Cabanac (1971, 1979) provided one of the most extensive examinations of the interaction between internal state and taste processing. In psychophysical studies, humans rated the pleasure and intensity of taste stimuli when hungry or sated. Changes in internal state produced no alterations in stimulus intensity ratings. In contrast, the affective (or pleasurable) component of gustatory stimuli was reduced when sated compared to when hungry (Cabanac \& Duclaux, 1970a; Cabanac, Duclaux \& Spector, 1971; Scherr \& King, 1982). Cabanac suggested the term "alliesthesia" to refer to the change in taste reactivity with nutritional state.

Alliesthesia is bidirectional. Positive and negative shifts in the pleasure derived from taste cues can be produced. Negative alliesthesial refers to a reduced affective response to taste cues and is observed after a meal or intragastric or intraduodenal loads (Cabanac \& Duclaux, 1970a; Cabanac et al., 1971; Scherr \& King, 1982). Positive alliesthesia, an enhanced positive response to tastes, is associated with hunger (Grill \& Norgren, 1978) or can be induced experimentally by eliciting an energy 
depletion state (Briese \& Quijada, 1979; Flynn \& Grill, 1983; Thompson \& Campbell, 1977).

The change in sensory responsivity with alliesthesia is restricted to the nutritionally-relevant stimulus. Glucose loads decrease the affective response to the taste of glucose but not to the taste of sodium chloride (Cabanac \& Duclaux, 1970a). Saline loads decrease the affective response to the taste of sodium chloride but not to glucose (Cabanac \& Duclaux, 1970a). Similarly, the consumption of a meal produces alliesthesia for food-related odors but not for food non-related odors (Duclaux, Feisthauer \& Cabanac, 1973).

The degree of alliesthesia depends on the organism's body weight history. Obese subjects tend not to show negative alliesthesia (Cabanac \& Duclaux, 1970b). Nornalweight subjects do not exhibit negative alliesthesia after weight loss but alliesthesia is restored when weight returns to pre-dieting levels (Cabanac et al., 1971).

Alliesthesia indicates that, in humans, internal state modulates the pleasantness of food tastes. Although the physiological signals producing alliesthesia are unidentified, alliesthesia is apparent when the organism is in a postprandial state produced by ingestion of a meal (Duclaux et al., 1973) or glucose solution (Cabanac et al., 1971). It seemed reasonable to us that the attenuated responsivity to taste characteristic of negative 
alliesthesia might contribute to spontaneous meal termination. Specifically, if the affective value of a food is reduced during the meal by the accumulating postingestive cues, organisms might be more likely to cease eating. This hypothesis provides a different perspective on satiety than the one currently dominant in physiological psychology, that is, the search for a unique set of satiety cues generated specifically to signal meal termination. The hypothesis that negative alliesthesia may contribute to meal termination is encouraged by correlations between properties of alliesthesia and satiety. For example, both satiety (Rolls, 1986) and alliesthesia show elements of sensory specificity. Also, food deprivation or body weight loss are frequently associated with increased eating and these manipulations attenuate or eliminate negative alliesthesia (Cabanac et al., 1971).

The experiments presented in this thesis address the hypothesis that a diminished affective response to taste provoked by the postprandial state contributes to meal termination. If this speculation is correct, then one should be able to demonstrate a reduced ability of taste cues to drive ingestion as postingestive consequences of food accumulate. I tested this prediction by evaluating whether a postprandial state reduces the excitatory effects of taste on eating. To isolate taste-driven intake, the sham feeding preparation (Weingarten \& Wastson, 1982) was 
employed. The postprandial state was experimentally controlled by injecting glucose ip. 


\section{EXPERIMENT 1}

Experiment 1 examines whether elevations of plasma glucose decrease eating in a preparation, sham feeding, in which intake is governed primarily by taste. This represents the most obvious examination of whether postabsorptive glucose diminishes the excitatory effect of taste on ingestion. The ability of manipulations such as cholecystokinin injection (Antin, Gibbs \& Smith, 1978; Forsyth, Weingarten \& Collins, 1985) or nutrient preloads (Antin, Gibbs \& Smith, 1977) to affect eating depends on the temporal relationship between the manipulation and the initiation of eating. Thus, we assessed the effects of ip glucose on sham feeding when it was injected either contiguous with, or remote from, meal initiation.

\section{Method}

Subjects were ten Long-Evans male rats weighing 330-430 grams at surgery. They were housed individually in hanging cages in a room maintained at $21 \mathrm{C}$ and on a 12:12 light:dark cycle. Food and water were available ad lib except as specified below. Each rat had a chronically indwelling gastric cannula implanted according to procedures detailed elsewhere (Weingarten \& Powley, 1980). 
Rats were tested when $3 \mathrm{hr}$ food deprived. Food was removed daily at 08:00. To ensure that all rats were $3 \mathrm{hr}$ deprived at testing, they were trained to drink $5 \mathrm{mls}$ of Carnation milk diluted 1:1 with water at the beginning of the deprivation period. Three hr after this procedure, each animal was prepared for sham feeding by opening its gastric cannula and cleaning its stomach with saline. Rats were trained to sham feed reliably before experimental treatments were begun. The liquid diet sham fid was $15 \%(\mathrm{w} / \mathrm{v})$ sucrose. Animals were tested in two conditions; the protocol is summarized in Table 1 . In the Paired condition, animals received a 5 min priming sham feed before ip injection. In the Non-Paired condition, rats simply waited in the test cages for $5 \mathrm{~min}$ prior to injection. In both conditions, a 5 min wait was interposed between ip injection and initiation of the test sham feed, which was 30 minutes in duration. Rats were injected with $\mathrm{D}$-glucose $(1.0$ or $1.5 \mathrm{~g} / \mathrm{kg})$ diluted with .15M saline to a total volume of $1 \mathrm{ml}$. The control injection was $1 \mathrm{ml} .15 \mathrm{M}$ saline. (Since rats weighed approximately $400 \mathrm{~g}$, in the maximal glucose injection, 1.5 $\mathrm{g} / \mathrm{kg}$, rats received about $2.4 \mathrm{kcals}$ of glucose. Under realfeeding conditions, rats would have consumed about 8.2 kcals of sugar). This protocol provided a total of 6 test conditions and rats were tested twice at each condition. The sequence of test conditions was determined randomly. 
Table 1. Protocol for experiment 1.

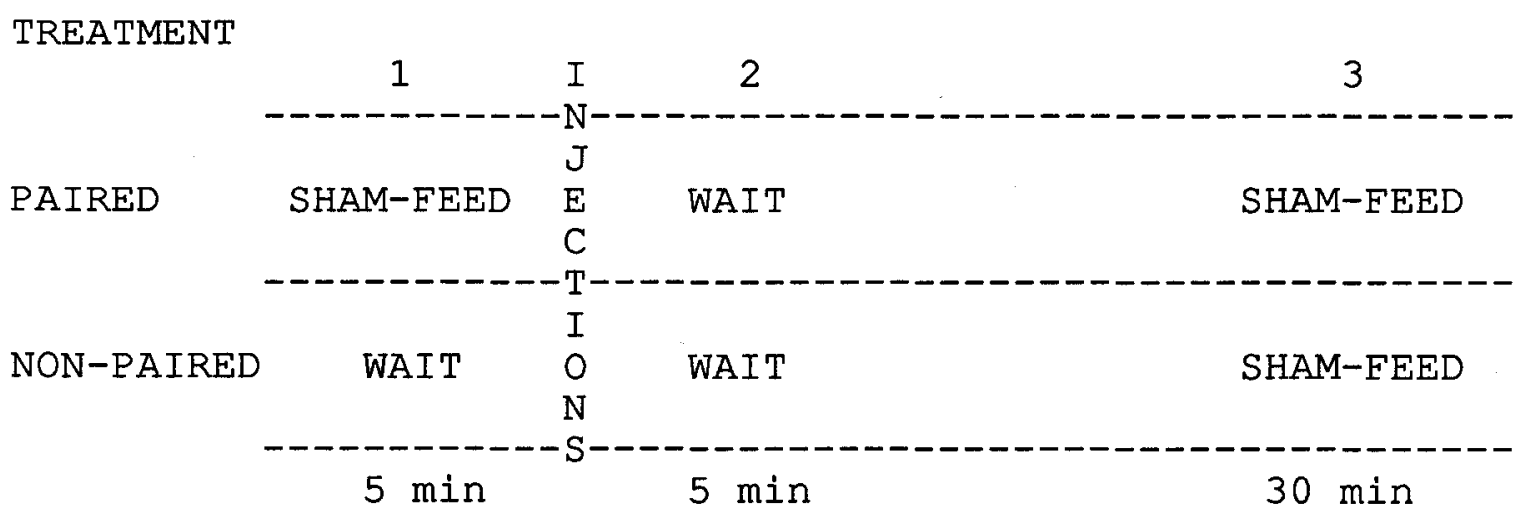


Results and discussion

Because 30 min intakes in the Paired and Non-Paired condition differed significantly even after ip saline, $\underline{E}(1,9)=10.238, \underline{p}<.01$, separate analyses of variance (ANOVA) were conducted for Paired and Non-Paired conditions. Cumulative intakes at 5, 15, and $30 \mathrm{~min}$ were analyzed. As shown in Figure 1, a significant dose-dependent reduction of intake was observed in the paired group at $5 \mathrm{~min}, \underline{\mathrm{F}}(2,18)=$ 16.186, $\underline{\mathrm{p}}<.001$ and $15 \mathrm{~min}, \underline{\mathrm{F}}(2,18)=17.526, \underline{\mathrm{p}}<.001$. By 30 min, no significant treatment effects were apparent, $F(2,18)=2.566$, NS. Post-hoc comparisons with Dunnett's test (Howell, 1982) indicated that the significant effects at 5 and $15 \mathrm{~min}$ resulted from a depressed intake after the $1.5 \mathrm{~g} / \mathrm{kg}$ dose of glucose $(\underline{p}<.01)$. The $1 \mathrm{~g} / \mathrm{kg}$ dose of glucose produced no significant inhibition of eating at any time point. In the Non-Paired condition, the only significant suppression of eating occurred $5 \mathrm{~min}$ after injection of $1.5 \mathrm{~g} / \mathrm{kg}$ glucose, $\underline{\underline{F}}(2,18)=4.868, \underline{p}<.05$. This effect was transitory, however, as no significant differences were found at $15 \mathrm{~min}, E(2,18)=1.571$, NS. Also, the magnitude of suppression with $1.5 \mathrm{~g} / \mathrm{kg}$ glucose at 5 min in the Non-Paired condition was only one-half that seen in the Paired condition (21\% Vs. 42\%).

The result that ip glucose suppresses sham feeding suggests that elevated plasma glucose reduces the ability of taste sensations to drive ingestion. The amount of sham 
Figure 1. Cumulative amount of $15 \%$ sucrose sham fed in Paired (left) and Non-Paired (right) treatment conditions. 
Paired Treatment

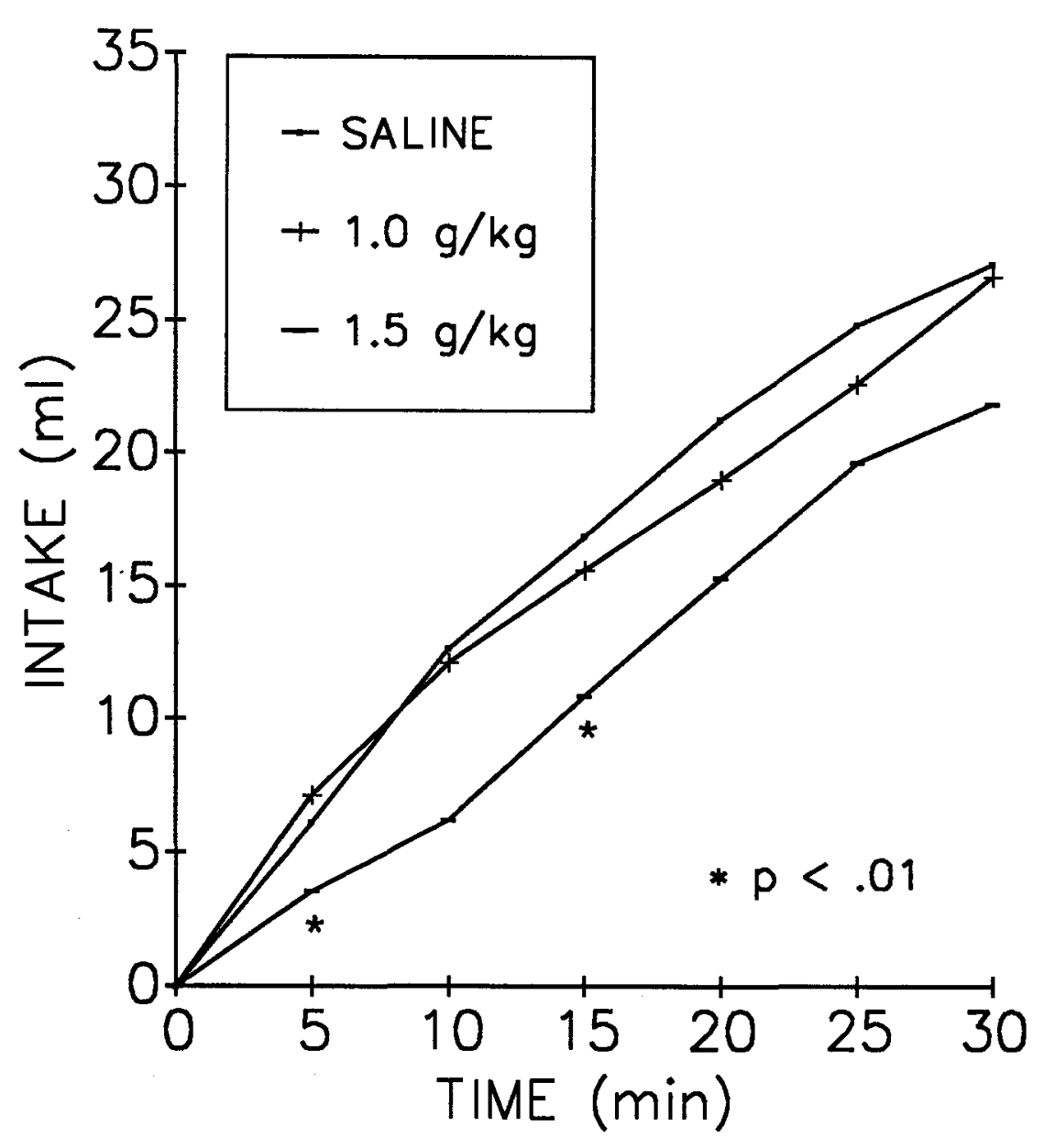

Non-Paired Treatment

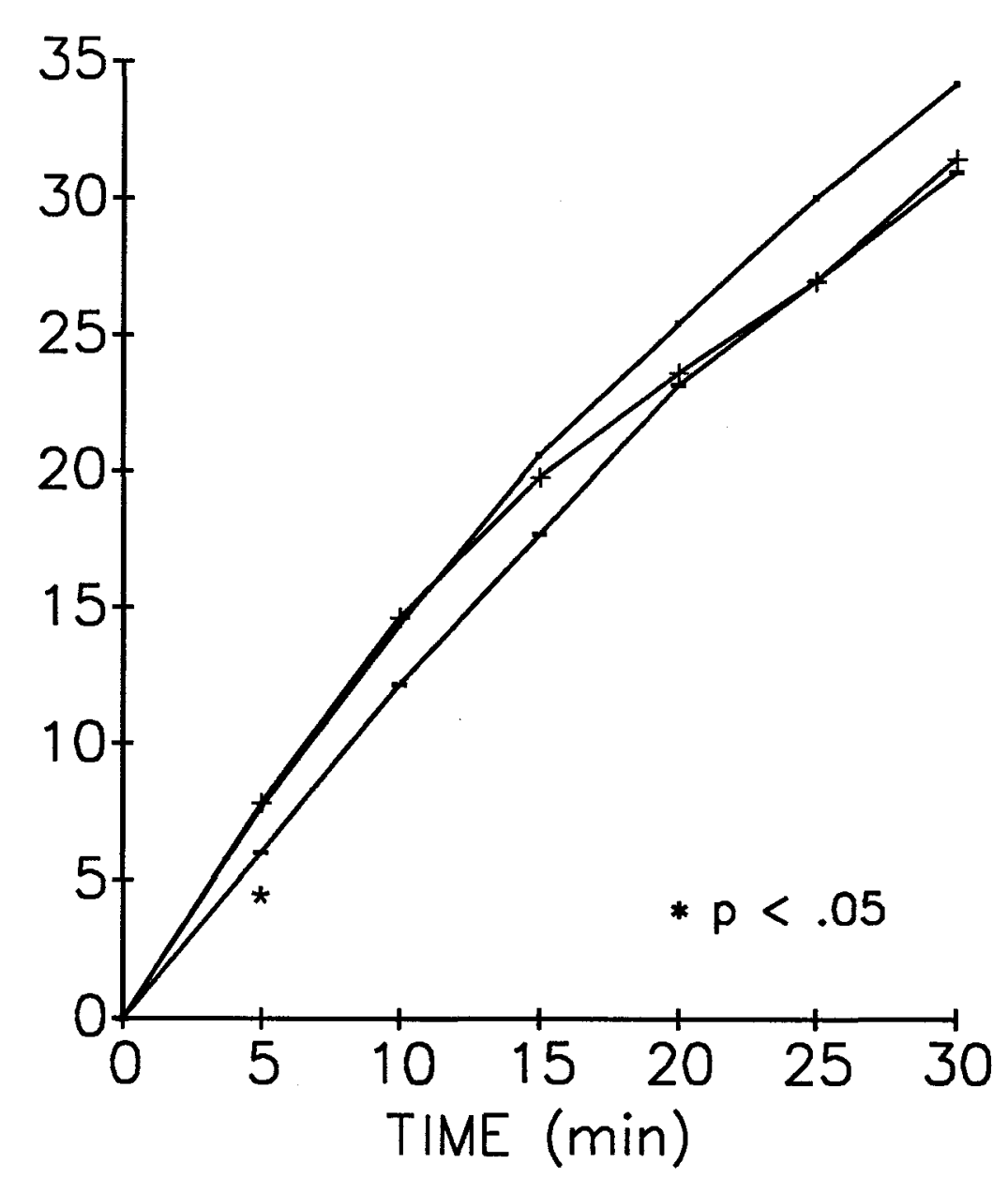


feeding inhibition produced by glucose injection, a maximum percent suppression of $42 \%$ at $5 \mathrm{~min}$ and $36 \%$ at $15 \mathrm{~min}$ (in the Paired condition) is similar to the maximal inhibition of sham feeding produced by manipulations such as exogenous cholecystokinin (Forsyth et al., 1985).

The heightened glucose-induced inhibition in the Paired, compared to the Non-Paired, condition highlights the importance of context in determining the ability of peripheral signals to induce satiety. The satiety produced by CCK (Antin et al., 1978; Forsyth et al., 1985; Gosnell\& Hsiao, 1981), gastric loads (Baile, Zinn \& Mayer, 1971), and duodenal loads (Antin et al., 1977), synergizes with oropharyngeal stimulation. The present results indicate similar oropharyngeal synergy with postabsorptive glucose.

It is unlikely that the glucose-induced inhibition of sham feeding results from malaise. First, no sustained reduction of eating is observed when the glucose is injected unpaired with contiguous oropharyngeal stimulation. If malaise were the mechanism underlying glucose-induced suppression, it should be as readily apparent, and of similar magnitude, in the Non-Paired and Paired condition. Second, if a manipulation causes malaise, one often observes a generalized reduction of intake on subsequent test days. However, statistical analyses revealed that there were no differences in the amounts eaten in the priming sham feeds in the different test conditions, $F(2,18)=0.82$, NS. In 
fact, analysis of the amounts eaten in the priming sham feeds on days following $1.5 \mathrm{~g} / \mathrm{kg}$ glucose revealed no significant differences, $F(1,9)=2.709$, NS, compared to days following saline. 
EXPERIMENT 2

The previous experiment demonstrates that $1.5 \mathrm{mg} / \mathrm{kg}$ glucose injected ip contiguous with oral stimulation suppresses sham feeding. The absence of a similar suppression when the identical injection is given unpaired with oropharyngeal stimulation suggests that this eating inhibition is unlikely to result from malaise. However, the possibility that malaise, or some nonspecific effect of the injection, produces the inhibition of eating is conceivable any time a manipulation results in a decrement of behavior. Thus, I conducted two experiments to determine whether the suppression of eating described in Experiment 1 results from the osmotic or malaise-inducing properties of the injection.

Method, results and discussion

First, I examined whether $1.5 \mathrm{mg} / \mathrm{kg}$ glucose ip would suppress any ongoing behavior. Ten rats were adapted to a $23 \mathrm{hr}$ water deprivation schedule for 6 days. On these days, immediately before access to water, rats were injected ip with $1 \mathrm{ml} .15 \mathrm{M}$ saline. On the test day, rats were injected ip with $1.5 \mathrm{mg} / \mathrm{kg}$ glucose. In addition to monitoring water intake, I also measured the amount of Purina Laboratory Rodent Pellets eaten in $1.5 \mathrm{hr}$ beginning with access to the water. 
Glucose, $1.5 \mathrm{mg} / \mathrm{kg}$ ip, did not suppress water drinking in water-deprived rats. The left panel of Figure 2 shows the amount of water rats drunk in $60 \mathrm{~min}$ when they were injected with saline or $1.5 \mathrm{mg} / \mathrm{kg}$ glucose. The difference in consumption between these days is not significant, $t(9)=$ .20 , NS. However, $1.5 \mathrm{mg} / \mathrm{kg}$ glucose did affect the amount rats ate during the time they had access to water. Glucose ip reduced significantly the amount rats ate in that time, $t(9)=5.73, p<.001$. These data are shown in the right panel of Figure 2 .

Second, I used the biologically inactive form of glucose, L-glucose (Sigma, St. Louis, MO), to test whether the osmotic properties of the glucose injections accounted for its eating inhibitory effects. This procedure represents the ideal control for osmolarity-induced malaise since L-glucose is identical in osmotic properties to Dglucose yet possesses no biological activity. Ten rats were fitted with chronic gastric cannulae and tested according to the Paired sham feeding protocol of Experiment 1 . Once intakes with saline ip injections had stabilized, a sequence of test days was conducted to replicate the suppression of sham feeding with biologically-active glucose, D-glucose, and to assess the effects of the biologically-inactive glucose enantiomer, L-glucose. The dose used was $1.5 \mathrm{~g} / \mathrm{kg}$ and injections were made up to a total volume of $1 \mathrm{ml}$ with $.15 \mathrm{M}$ saline. 
Figure 2. Effect of $1.5 \mathrm{mg} / \mathrm{kg} \mathrm{D}$-glucose injected ip on water (left panel) and food (right panel) intake in water-deprived rats. Glucose suppressed food, but not water, intake. 
Water Intake

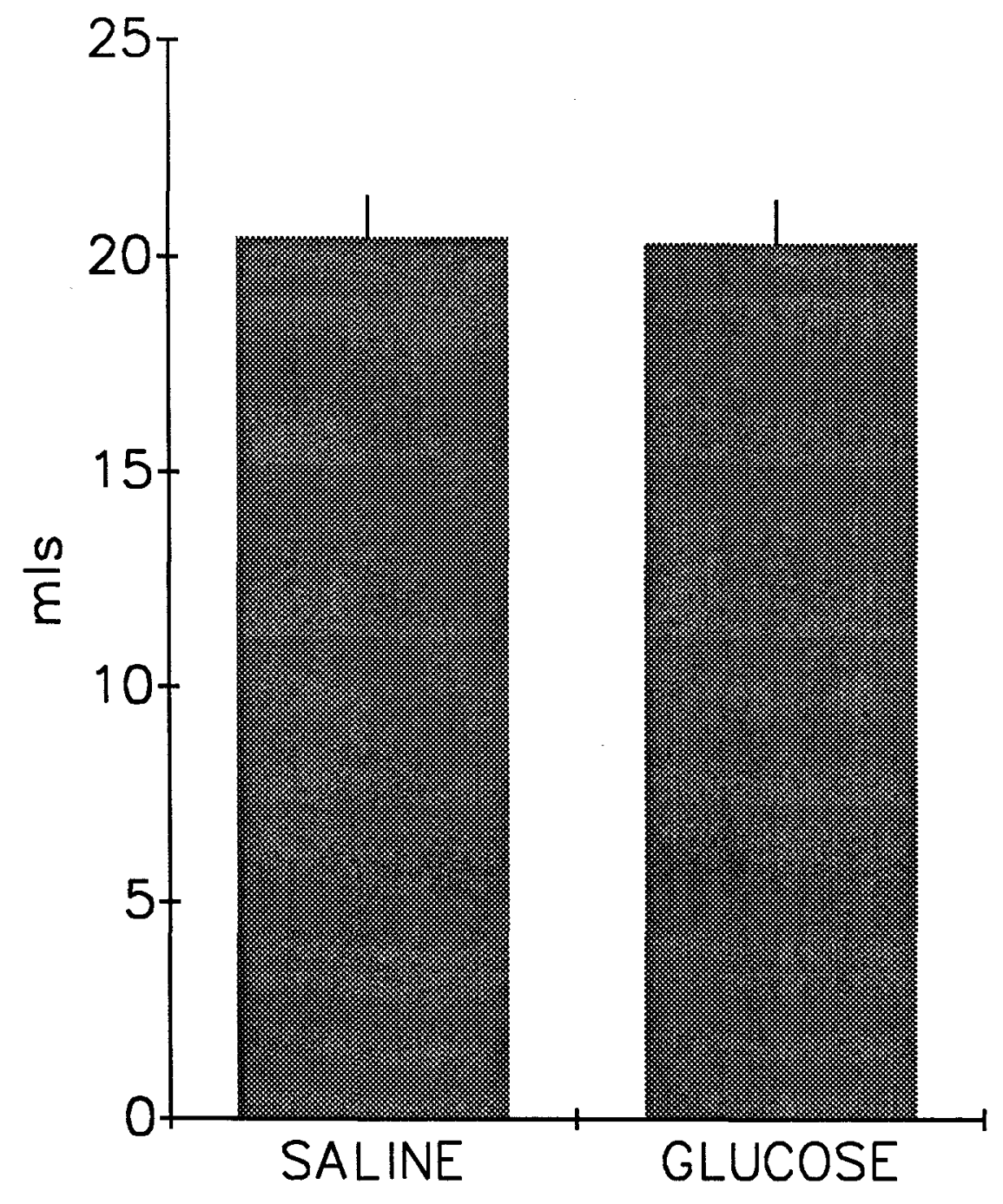

Food Intake

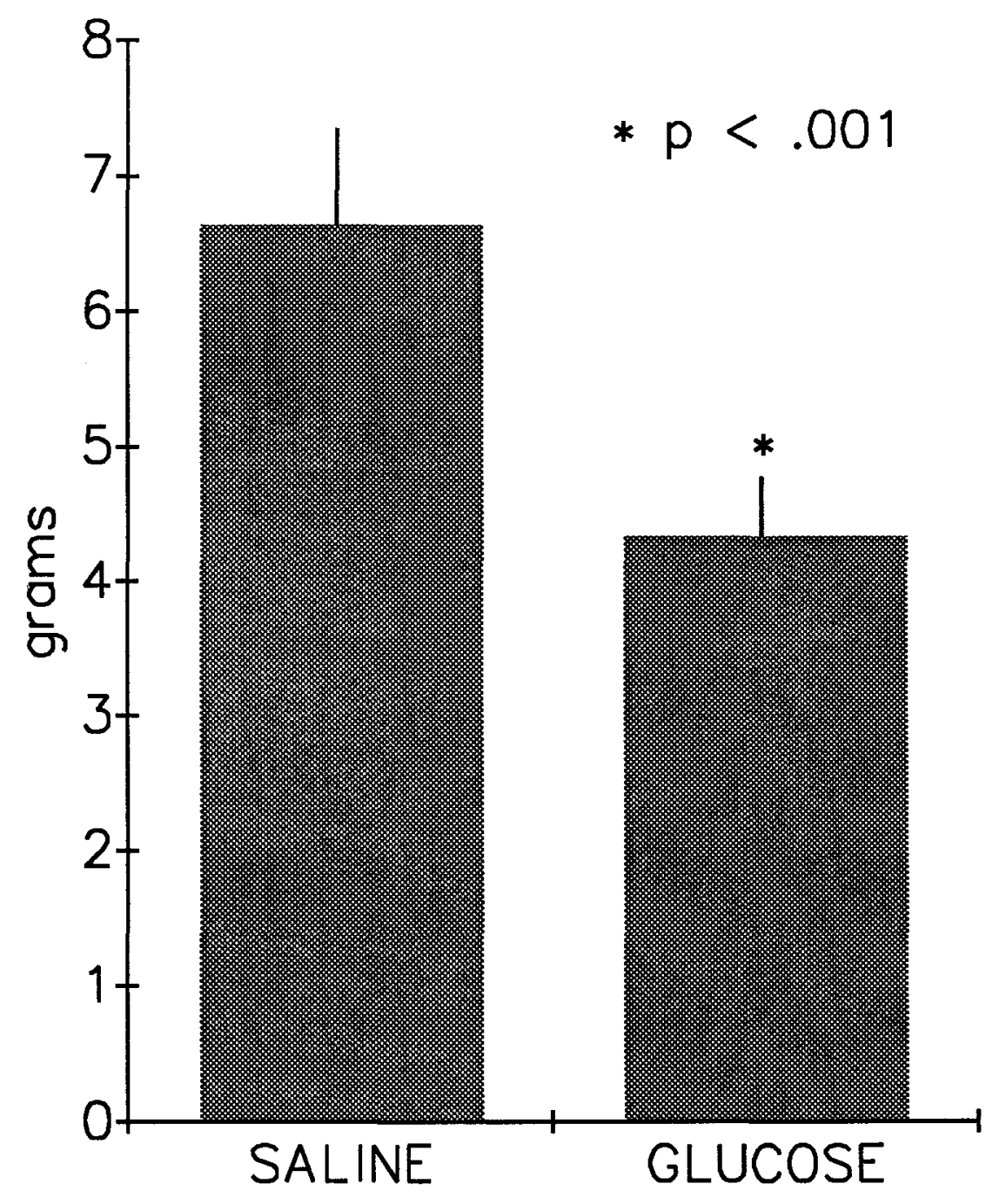


The results, presented in Figure 3, demonstrate that the reduction of sham feeding produced by glucose does not result from the osmotic properties of the injection. Consistent with Experiment 1, $1.5 \mathrm{mg} / \mathrm{kg}$ D-glucose significantly suppressed sham feeding compared to ip saline at $15 \mathrm{~min}, \underline{q}_{3}=5.82, \underline{\mathrm{p}}<.01$ and $30 \mathrm{~min}, \underline{q}_{3}=7.54, \underline{\mathrm{p}}<$ .01 . In contrast, with equivalent doses of L-glucose, animals showed no decrement of sham feeding at either $15 \mathrm{~min}$ $\underline{q}_{2}=2.07$, NS, or $30 \mathrm{~min}, \underline{q}_{2}=2.65$, NS. At both times, however, animals ate significantly less after D-glucose than L-glucose ( $\underline{\mathrm{p}}<.05$ at $15 \mathrm{~min}$ and $\underline{\mathrm{p}}<.01$ at $30 \mathrm{~min})$. These results make it unlikely that the suppression of sham feeding observed with D-glucose results from malaise or some nonspecific effect of the injection. The dose of glucose which is capable of suppressing sham feeding, 1.5 $\mathrm{mg} / \mathrm{kg}$, does not inhibit water drinking in thirsty animals yet, under identical experimental circumstances, suppresses eating. L-glucose, which shares almost all of the physical properties of D-glucose but which is without its biological activity, does not produce any inhibition of eating. Thus, the inhibition of sham feeding with ip glucose appears to represent a postabsorptive attenuation of the ability of taste cues to drive ingestion. 
Figure 3. Effect of the L- and D- enantiomers of glucose injected ip on cumulative amounts sham fed 15 and 30 minutes after meal initiation. D-glucose suppressed intake; I-glucose did not. 
Amount of $15 \%$ Sucrose Sham Fed

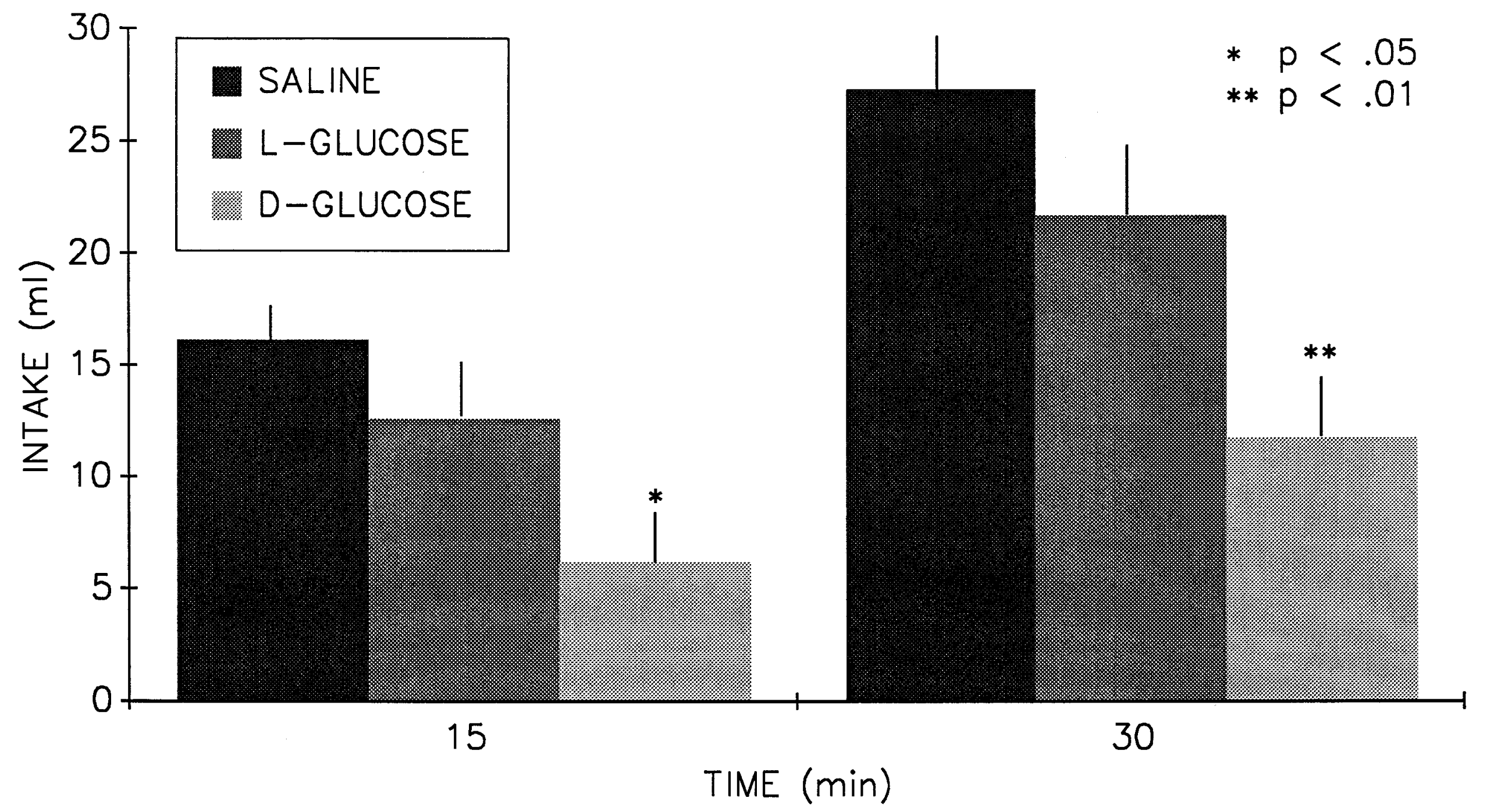


EXPERIMENT 3

The results of Experiment 1 suggest an interaction between a postabsorptive glucose-related satiety signal and oropharyngeal stimulation. The present experiment examines the temporal nature of this synergy in more detail.

\section{Method}

The animals used in Experiment 1 were tested at various test conditions differing with respect to ip injection administered (.15M saline or $1.5 \mathrm{~g} / \mathrm{kg}$ glucose) and when the injection was administered relative to the initiation of the test sham feed. Injections were given $5 \mathrm{~min}$ before the start of sham feeding $(-5 \mathrm{~min})$, at the start of sham feeding ( $0 \mathrm{~min}), 5 \mathrm{~min}(+5 \mathrm{~min})$, or $10 \mathrm{~min}(+10 \mathrm{~min})$, after sham feeding had begun. Animals were tested twice at each of the 8 experimental conditions.

Results and discussion

The temporal relationship between ip glucose and oropharyngeal stimulation was an important determinant of the amount sham fed. The amounts rats sham fed in the 5 minutes after ip injection of glucose or saline is shown in Table 2. Glucose injections given $5 \mathrm{~min}$ before the initiation of sham feeding did not affect amount eaten. A 
Table 2. Effect of $1.5 \mathrm{~g} / \mathrm{kg}$ glucose or .15M saline injected ip on amount sham fed in the 5 minutes subsequent to the injection. Data indicated are absolute intakes. Injection Time indicates the time (in min) the injection was given. Time 0 is the initiation of the sham feed.

Injection

\begin{tabular}{ccc} 
Injection Time & Saline & Glucose \\
\hline-5 & 7.95 & 6.90 \\
0 & 7.60 & 4.70 \\
+5 & 6.95 & 2.45 \\
+10 & 5.55 & 2.15
\end{tabular}


suppressive effect was observed only when glucose was administered coincident with, or after, initiation of sham feeding. Since the absolute intakes following injections administered later in the sham feeding bout $(e . g .,+10 \mathrm{~min})$ were lower than those with early injections (e.g., $-5 \mathrm{~min}$ ) I compared the amount with which glucose inhibited sham feeding by expressing intakes following ip glucose as a ratio of intakes in the relevant saline control condition. These percent suppressions were defined as: 1-(intake after glucose/intake after saline) $\mathrm{x}$ 100. The mean percent suppressions at $5 \mathrm{~min}$ postinjection are graphed in Figure 4 and showed that sham feeding inhibition increased significantly with amount of oropharyngeal stimulation prior to the injection $\underline{\underline{F}}(3,27)=10.612$, $\underline{\mathrm{p}}<.001$.

These results indicate that the potency with which ip glucose suppresses sham feeding depends on when it is injected relative to the feeding bout. These data complement previous demonstrations of synergy between oropharyngeal stimulation and satiety manipulations (Antin et al., 1977, 1978; Baile et al., 1971; Forsyth et al., 1985; Gosnell \& Hsiao, 1981). These results also strengthen the suggestion that ip glucose does not inhibit eating via malaise. Glucose injected after a $5 \mathrm{~min}$ bout of feeding produces more inhibition of eating than the identical injection given coincident with the initiation of eating. If malaise were the factor responsible for the inhibition, a 
Figure 4. Percent suppression observed after $1.5 \mathrm{~g} / \mathrm{kg}$ glucose. Oropharyngeal stimulation increases the potency with which ip glucose suppresses eating. 
\% Suppression After IP Glucose $(1.5 \mathrm{~g} / \mathrm{kg}$ )

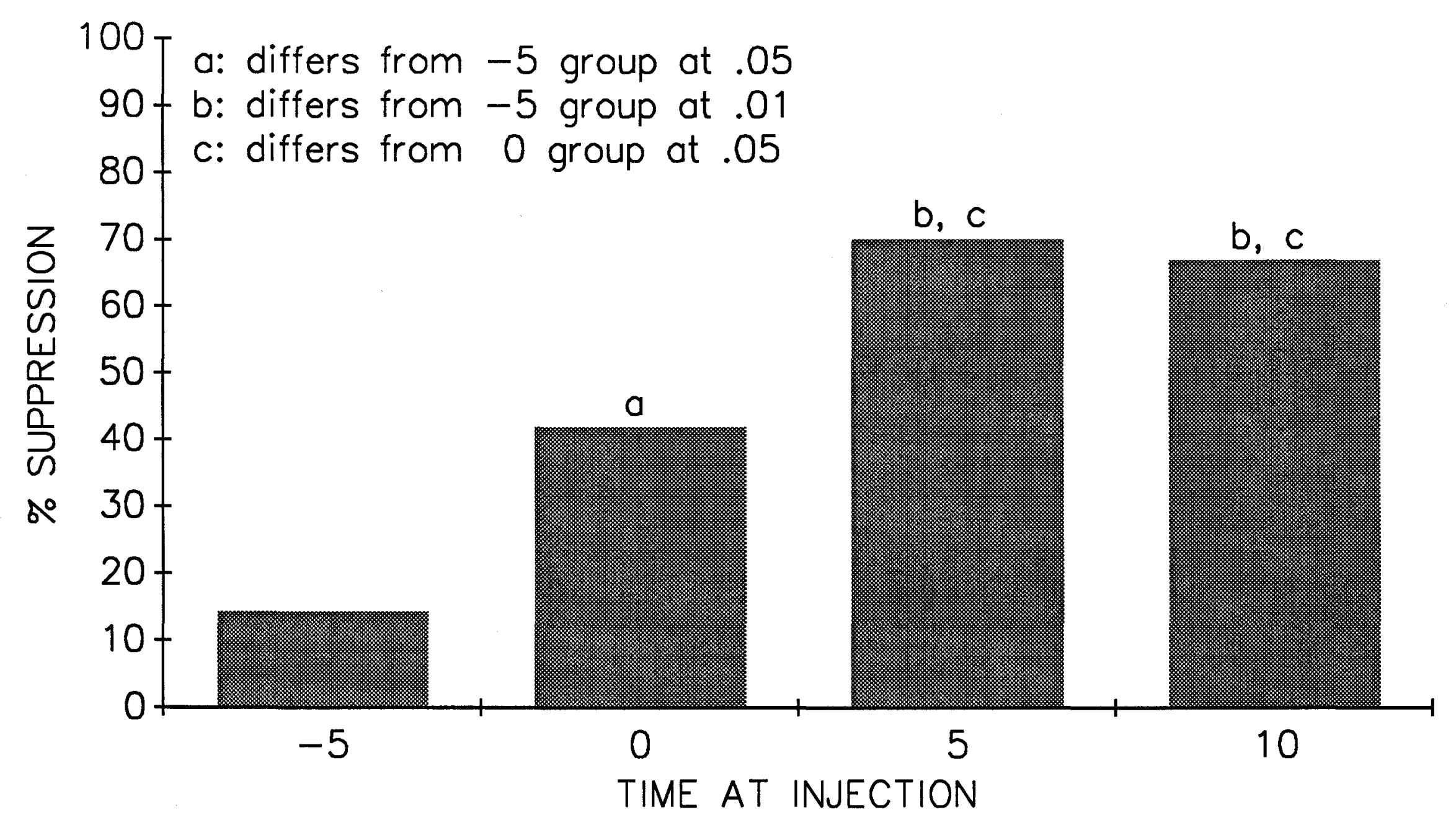


similar amount of intake reduction would be expected and this is not observed. 


\section{EXPERIMENT 4}

A significant suppression of sham feeding has been demonstrated only at the $1.5 \mathrm{~g} / \mathrm{kg}$ dose. The ability of ip glucose to suppress eating should depend on the drive to eat and, in the present experimental circumstances, motivation is high since rats are sham feeding a highly palatable solution (Sclafani \& Nissenbaum, 1985; Weingarten \& Watson, 1982). The present data examine the relationship between diet palatability and the satiety produced by ip glucose. Specifically, I determine if decreasing the sucrose concentration of the diet diminishes the dose of glucose necessary to produce significant suppressions of sham feeding .

\section{Method}

The protocol is identical to the Paired condition in Experiment 1. Briefly, rats had a 5 min priming sham feed. Then, they were injected ip with either $1 \mathrm{ml}$ of $.15 \mathrm{M}$ saline or glucose in doses of $0.5,1.0$ or $1.5 \mathrm{~g} / \mathrm{kg}$ made up to a volume of $1 \mathrm{ml}$. After a $5 \mathrm{~min}$ wait, rats were given access to sucrose for a $30 \mathrm{~min}$ test sham feed. The concentrations of sucrose in the test sham feed were $5 \%$, $10 \%$ or $15 \%$ sucrose. To eliminate contrast effects, the concentration 
of sucrose in the priming sham feed was identical to that offered in the test sham feed.

Results and discussion

The absolute intakes in the test conditions are shown in Table 3. Since the absolute amount sham fed varies with sucrose concentration (Weingarten \& Watson, 1982) I expressed the amount by which ip glucose suppressed intake as a percent suppression relative to the relevant control. These data are also presented in Table 3 .

In general, the amount of suppression produced by ip glucose depended on the sucrose concentration of the diet and the dose of glucose. A two-factor ANOVA indicated that the amount of intake suppression increased significantly with increasing glucose dose, $\underline{E}(2,56)=5.901$, $\underline{p}<.005$. In addition, the magnitude of suppression increased significantly as the concentration of the sucrose decreased $\underline{E}(2,56)=3.299, \underline{p}<.05$. Thus, smaller doses of ip glucose inhibited less palatable sucrose solutions. 
Table 3. The effect of ip glucose on amount sham fed.

"Abs" indicates the absolute amount eaten (in $\mathrm{m} / \mathrm{s}$ ) in the test condition. "을 indicates the percent suppression of sham feeding produced by ip glucose at doses indicated. Percent suppression is calculated as: 1-(intake after glucose/intake after saline) $\times 100$. Negative percent suppressions indicate greater consumption after saline than glucose.

Sucrose concentration ( $w / v)$

Glucose dose

$\underline{5 ㅇ ㅜ ㅇ ~}$

$10 \frac{\circ}{2}$

$15 \%$

Abs $\frac{8}{8} \mathrm{~S}$

Abs $\frac{8}{8} \mathrm{~S}$

Abs $\div \mathrm{S}$

Saline

7.3

14.1

19.8

$0.5 \mathrm{~g} / \mathrm{kg}$

$5.4 \quad 17 \%$

$14.4-2 \%$

$19.3 \quad 3 \%$

$1.0 \mathrm{~g} / \mathrm{kg}$

$5.3 \quad 27 \%$

$12.0 \quad 11 \%$

$18.7 \quad 7 \%$

$1.5 \mathrm{~g} / \mathrm{kg}$

$3.646 \%$

$9.437 \%$

$15.6 \quad 19 \%$ 
EXPERIMENT 5

The motivation behind the current study was to investigate whether reductions in the affective value of hedonically positive taste stimuli could contribute to meal termination. The experiments presented so far have employed sham feeding to isolate the effects of ip glucose on tastedriven food intake. The finding that ip glucose inhibits sham feeding is consistent with the idea that postabsorptive glucose may modulate taste reactivity. For these findings to be relevant to satiety, however, similar findings must be evident in normally feeding animals. Data obtained in the sham feeding preparation do not always generalize to normal feeding conditions (Black \& Weingarten, 1989; Nissenbaum \& Sclafani, 1988). Furthermore, the effects of plasma glucose manipulations on real eating have been equivocal. Some report an inhibition of eating with glucose administration (Rowland \& Stricker, 1979; Smith, 1966). Others report no effect (Baile et al., 1971; Yin \& Tsai, 1973). And, still others report an increase (Geiselman \& Novin, 1982). The type of glucose manipulation and other particulars of the test situation appear to determine whether, and how, glucose influences eating. Therefore, the present study investigates the satiety effect of ip glucose in real 
feeding animals under test conditions used in previous experiments.

\section{Method}

To eliminate satiety induced by a priming sham feed, these studies mimicked the Non-Paired condition in Experiment 1. Briefly, animals were injected with glucose ip at doses of $0.5,1.0$, or $1.5 \mathrm{~g} / \mathrm{kg} 5 \mathrm{~min}$ before the initiation of a test meal of $15 \%$ sucrose available for 30 min. The control injection was .15M saline. Rats in this study maintained their gastric cannula closed to permit real feeding .

Results and discussion

As shown in Figure 5, glucose produced a dose-dependent inhibition of eating. A significant glucose-induced suppression was evident at $5 \mathrm{~min}, \mathrm{~F}(3,24)=8.746, \mathrm{p}<.001$, $15 \mathrm{~min}, \underline{\mathrm{F}}(3,24)=5.934, \underline{\mathrm{p}}<.005$ and $30 \mathrm{~min} F(3,24)=$ $5.667, \underline{p}<.005$. A maximal 55\% suppression of meal size was observed after $1.5 \mathrm{~g} / \mathrm{kg}$ glucose $5 \mathrm{~min}$ postinjection. Multiple comparisons were conducted with Dunnett's test to permit comparisons between experimental and control conditions. Animals ate significantly less following 1.0 (p $<.05)$ and $1.5 \mathrm{~g} / \mathrm{kg}(\underline{\mathrm{p}}<.01)$ glucose compared to saline. The $0.5 \mathrm{~g} / \mathrm{kg}$ glucose dose produced no significant reduction of eating. 
Figure 5. Cumulative amount of $15 \%$ sucrose eaten by animals with cannulae closed. Both 1.0 and $1.5 \mathrm{~g} / \mathrm{kg}$ doses significantly reduced intake. 


\section{Cumulative $15 \%$ Sucrose Intake}

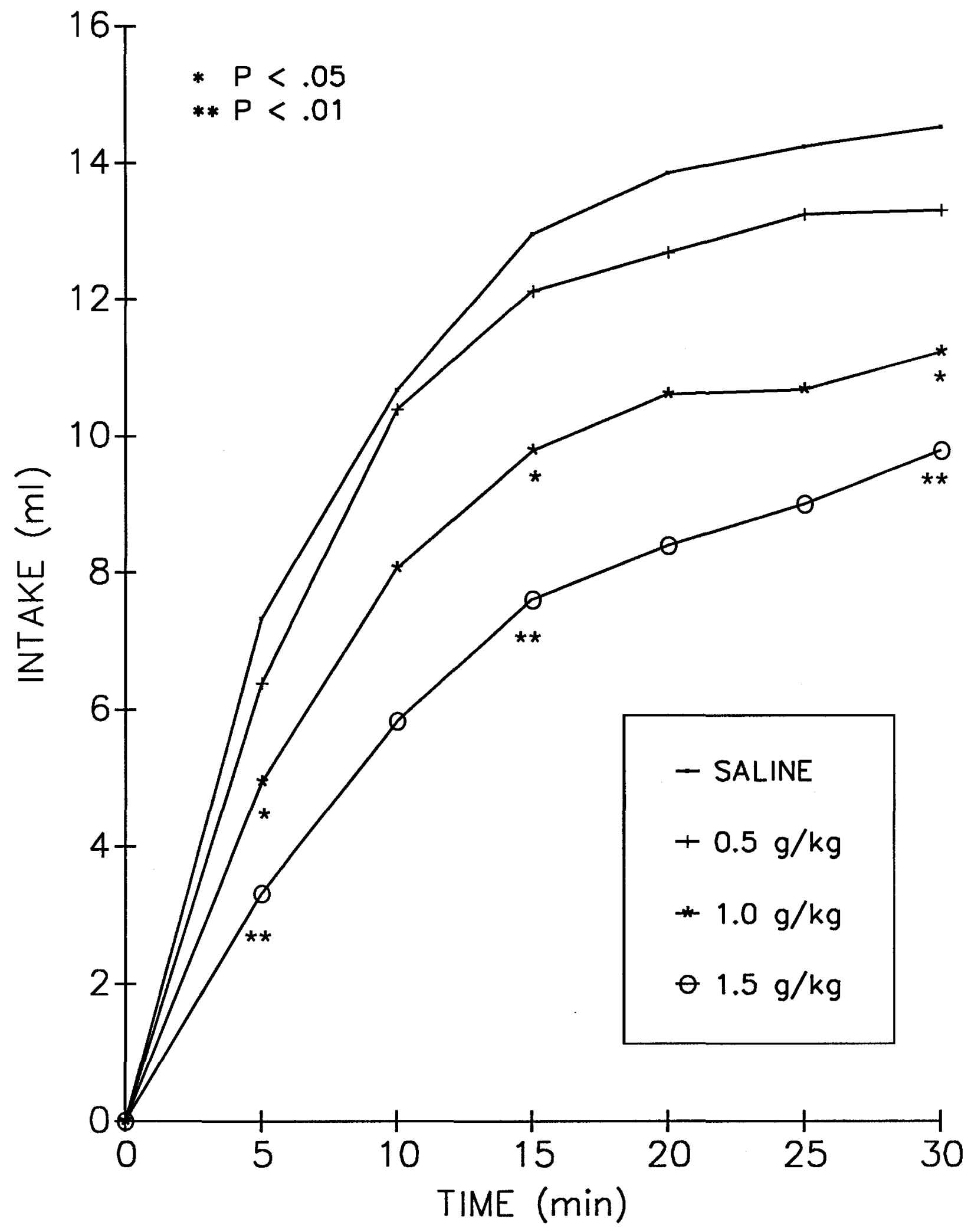


Glucose injection had a greater suppressive effect on real compared to sham feeding. For example, $1.0 \mathrm{~g} / \mathrm{kg}$ glucose 15 min after injection resulted in only a $7 \%$ suppression of sham feeding in the Paired condition of Experiment 1. The identical dose, at the identical time, produced a $24 \%$ suppression of real feeding. Because real feeding activates a host of peripheral satiety mechanisms not elicited by sham feeding, this potentiated response to ip glucose most likely represents the interaction of ip glucose with postgastric satiety signals. 


\section{GENERAL DISCUSSION}

This study examined whether postabsorptive glucose affects the ability of taste cues to motivate food intake. I manipulated postabsorptive glucose by administering glucose ip and isolated the effects of taste on intake by using sham feeding. Elevated glucose attenuated the ability of hedonically positive tastes to motivate ingestion. The ability of ip glucose to inhibit sham feeding synergizes with both oropharyngeal stimulation and other satiety signals emanating from the gastrointestinal tract. Also, the degree to which ip glucose inhibits eating depends upon the stimulus properties of the diet being consumed; less preferred diets are inhibited by smaller doses of ip glucose. Sham feeding of concentrated sugar solutions leads sometimes to small elevations of plasma glucose which appear not to affect amount eaten (Sclafani \& Nissenbaum, 1985). The suppressive effect of glucose on sham feeding observed in the present experiments suggests that the dynamics, magnitude, or mode of delivery of glucose or the maintenance body weight of the animal are important in whether postabsorptive glucose rises will inhibit eating.

The synergy of ip glucose and oropharyngeal stimulation is congenial with similar findings of synergy between oropharyngeal cues and other satiety-producing manipulations 
such as CCK injection (Antin et al., 1978; Forsyth et al., 1985; Gosnell \& Hsiao, 1981) or intraduodenal infusions (Antin et al., 1977). These demonstrations make it clear that the context in which peripheral signals are elaborated is critical to whether they produce satiety or not. Manipulations which inhibit eating often fail to do so if they are present in the absence of an appropriate behavioral context (e.g., Forsyth et al., 1985) and oropharyngeal stimulation appears to provide a context in which the satiety action of such events is expressed. The mechanism by which oropharyngeal stimulation potentiates the satiety effect of peripheral events is unclear. Oral stimulation may actually alter the physiological effect of the peripheral manipulation to enhance or permit its satiety effect. For example, oropharyngeal stimulation in a glucose tolerance test alters the absorption and glycemia profile of ingested glucose compared to identical glucose loads administered intravenously. Alternatively, oropharyngeal stimulation may potentiate satiety by altering the interpretation of peripheral signals. That is, the physiological action of a manipulation such as ip glucose or CCK injection may be identical in the presence or absence of oropharyngeal stimulation. However, oropharyngeal stimulation might alter the brain's interpretation of that event. It is unclear which of these interpretations is correct. 
Electrophysiological data suggest one way that postabsorptive glucose might alter the ability of taste cues to drive ingestion. Activity evoked in the nucleus of the solitary tract (NST) in response to tastes applied to the tongue is decreased by elevations of plasma glucose (Giza \& Scott, 1983). Activity in the NST elicited by sucrose applied to the tongue is also inhibited by gastric distension (Glenn \& Erickson, 1976). Thus, ip glucose (and perhaps other peripheral satiety manipulations) might attenuate taste-driven intake by directly modulating activity in early order neurons processing gustatory information.

It is unclear which other peripheral or central sites mediate the feeding inhibitory effects of ip glucose. When glucose is administered ip, several feeding-related mechanisms are affected. There are, presumably, increases in portal glucose levels and, therefore, the amount of nutrient available to the liver. Hepatic mechanisms may be critical to the suppression of eating following ip glucose. Alternatively, the suppression of taste-driven intake may result from plasma hyperglycemia detected by central glucoreceptors.

Although the present results demonstrate clearly that ip glucose can reduce the motivating properties of taste, it is unclear to what degree this phenomenon contributes to spontaneous postprandial satiety. The present results 
provide an animal analogue to Cabanac's alliesthesia but, like his initial demonstrations, the relevance of this phenomenon to spontaneous satiety is unclear. 


\section{EOOTNOTE}

1 One interpretation of "negative alliesthesia" is controversial. Some have taken it to indicate that tastes actually become aversive and this finding has not been obtained reliably. The term "negative alliesthesia" is used here to indicate simply that subjects report a reduction in the pleasantness of the taste and not that subjects find the tastes aversive. 
References

Antin, J., J. Gibbs and G.P. Smith. Intestinal satiety requires pregastric food stimulation. Physiol Behav 18: $421-425,1977$.

Antin, J., J. Gibbs and G.P. Smith. Cholecystokinin interacts with pregastric food stimulation to elicit satiety in the rat. Physiol Behav 20: 67-70, 1978. Baile, C.A., W. Zinn and J. Mayer. Feeding behavior of monkeys: Glucose utilization rate and site of glucose entry. Physiol Behav 6: 537-541, 1971.

Black, R.M. And H.P. Weingarten. A comparison of taste reactivity changes induced by ventromedial hypothalamic lesions and stria terminalis transections. Physiol Behav 44: 699-708, 1989.

Booth, D.A. Conditioned satiety in the rat. I Comp Physiol Psychol 81: 457-471, 1972 .

Booth, D.A. Food-conditioned eating preferences and aversions with interoceptive elements: Conditioned appetites and satieties. Ann NY Acad Sci 443: 22-42, 1985 .

Briese, E. and M. Quijada. Positive alliesthesia after insulin. Experientia 35: 1058-1059, 1979.

Cabanac, M. Physiological role of pleasure. Science 173: 1103-1107, 1971 .

Cabanac, M. Sensory pleasure. Quart Rev Biol 54: 1-29, 1979. 
Cabanac, M. and R. Duclaux. Specificity of internal signals in producing satiety for taste stimuli. Nature 227: 966-967, 1970a.

Cabanac, M. and R. Duclaux. Obesity: Absence of satiety aversion to sucrose. Science 168: 496-497, 1970b. Cabanac, M., R. Duclaux and N.H. Spector. Sensory feedback in regulation of body weight: Is there a ponderstat? Nature 229: 125-127, 1971.

Cagan, R.H. and O. Maller. Taste of sugars: Brief exposure single-stimulus behavioral method. J Comp Physiol Psychol 87: 47-55, 1974.

Christensen, K.R. Methodology in preference testing. In:

Physiological and Behavioral Aspect of Taste, edited by M.R. Kare and B.P. Halpern. Chicago: University of Chicago Press, 1961, pp. 79-91.

Davis, J.D. The effectiveness of some sugars in stimulating licking behavior in the rat. Physiol Behav 11: 39-45, 1973.

Davis, J.D. and C.S. Campbell. Peripheral control of meal size in the rat: Effect of sham feeding on meal size and drinking rate. J Comp Physiol Psychol 83: 379-387, 1973.

Davis, J.D. and M.W. Levine. A model for the control of ingestion. Psychol Rev 84: 379-412, 1977. 
Duclaux, R., J. Feisthauer and M. Cabanac. Effets du repas sur l'agrément d'odeurs alimentaires et nonalimentaires chez 1'homme. Physiol Behav 10: 1029-1033, 1973.

Flynn, F.W. and H.J. Grill. Insulin elicits ingestion in decerebrate rats. Science 221: 188-190, 1983. Forsyth, P.A., H.P. Weingarten and S.M. Collins. Role of oropharyngeal stimulation in cholecystokinin-induced satiety in the sham feeding rat. Physiol Behav 35: 539543,1985 .

Garcia, J., W. G. Hankins and K. W. Rusiniak. Behavioral regulation of the milieu interne in man and rat. Science $185,824-831,1974$.

Geiselman, P.J. and D. Novin. Sugar infusion can enhance feeding. Science 218: 490-491, 1982.

Giza, B.K. and T.R. Scott. Blood glucose selectively affects taste-evoked activity in rat nucleus tractus solitarius. Physiol Behav 31: 643-650, 1983.

Glenn, J.F. and R.P. Erickson. Gastric modulation of gustatory afferent activity. Physiol Behav 16: 561-568, 1976.

Gosnell, B. and S. Hsiao. Cholecystokinin satiety and orosensory feedback. Physiol Behav 27: 153-156, 1981. Grill, H.J. and K.C. Berridge. Taste reactivity as a measure of the neural control of palatability. In: progress in Psychobiology and Physiological Psychology, Vol. 11, 
edited by A.N. Epstein \& A.M. Sprague. Academic Press, New York, 1985, pp. 1-61.

Grill, H.J. and R. Norgren. Chronically decerebrate rats demonstrate satiation but not bait shyness. Science 201: 267-269, 1978 .

Howell, D.C. Statistical Methods for Psychology. Boston: PWS Publishers, 1982 .

Mook, D. G. Oral and postingestional determinants of the intake of various solutions on rats with esophageal fistulas. J Comp Physiol Psychol 56: 645-659, 1963. Nissenbaum, J.W. and A. Scalafani. A comparison of the effects of atropine on real-feeding and sham-feeding of sucrose in rats. Pharmacol Biochem Behav 29: 231-238, 1988 .

Riley, A.L. and D.L. Tuck. Conditioned food aversions: A bibliography. Ann NY Acad Sci 443: 381-438, 1985. Rolls, B.J. Sensory-specific satiety. Nutr Rev 44: 93-101, 1986.

Rowland, N. and E.M. Stricker. Differential effects of glucose and fructose infusions on insulin-induced feeding in rats. Physiol Behav 22: 387-389, 1979. Scherr, S. and K.R. King. Sensory and metabolic feedback in the modulation of taste hedonics. Physiol Behav 29: $827-832,1982$.

Sclafani, A. and J.W. Nissenbaum. On the role of the mouth and gut in the control of saccharin and sugar intake: A 
reexamination of the sham-feeding preparation. Brain Res Bull 14: 569-576, 1985.

Smith, M.H. Effects of intravenous injections on eating. J Comp Physiol Psychol 61: 11-14, 1966. Thompson, D.A. and R.G. Campbell. Hunger in humans induced by 2-deoxy-d-glucose: Glucoprivic control of taste preference and food intake. Science 198: 1065-1068, 1977 .

Weingarten, H.P. and T.L. Powley. A new technique for the analysis of gastric acid responses in the unanesthetized rat. Lab Anim Sci 30:673-680, 1980. Weingarten, H.P. and S.D. Watson. Sham feeding as a procedure for assessing the influence of diet palatability on food intake. Physiol Behav 28: 401-407, 1982 .

Yin, T.H. and C.T. Tsai. Effects of glucose on feeding in relation to routes of entry in rats. J Comp Physiol Psychol 85: 258-264, 1973.

Young, P.T. Palatability: the hedonic response to foodstuffs. In: Handbook of Physiology, section 6 , vol. 1, edited by C.F. Code. Baltimore: William and Wilkins, 1967, pp. 353-366. 